\title{
Considerations for Clinical Therapeutic Development of Statins for Neurodevelopmental Disorders
}

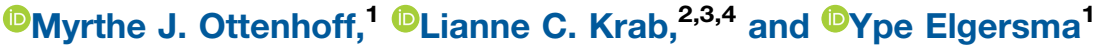

https://doi.org/10.1523/ENEURO.0392-19.2020

${ }^{1}$ Department of Neuroscience, ENCORE Expertise Center for Neurodevelopmental Disorders, Erasmus Medical Center, 3015 GD, Rotterdam, The Netherlands, ${ }^{2}$ Cordaan Outpatient Clinic for ID Medicine, 1033 PK, Amsterdam, ${ }^{3}$ Odion Outpatient Clinic for ID Medicine, 1531 AD, Wormer, and ${ }^{4}$ Department of Pediatrics, Amsterdam UMC, 1105 AZ, Amsterdam, The Netherlands

Key words: autism spectrum disorder; cognitive trials; fragile X; lovastatin, RASopathies; simvastatin

\section{Significance Statement}

The 3-hydroxy-3-methyl-glutaryl-coenzyme A reductase (HMG-CoA reductase) inhibitors lovastatin and simvastatin have both been investigated in clinical trials designed to treat the cognitive deficits associated with neurodevelopmental disorders such as neurofibromatosis type 1 , fragile $X$ and autism. In a recent study, the therapeutic efficacy of lovastatin and simvastatin were compared in a fragile $X$ ( $F m r 1$ ) mouse model. The authors concluded that lovastatin was superior to simvastatin in rescuing the Fmr1 phenotypes, and cautioned against considering simvastatin as treatment for neurodevelopmental disorders. We discuss these findings in the context of published literature and argue that more support is needed for this potentially far-reaching conclusion. We further provide recommendations to improve the translation of pre-clinical studies of cognitive disorders into the clinical domain.

The potential use of statins for antagonizing RAS (rat sarcoma viral oncogene homolog) signaling was first recognized nearly three decades ago (Mendola and Backer, 1990; Sebti et al., 1991). Functional RAS requires posttranslational farnesylation to become membrane bound and active. Since farnesyl (like cholesterol) is a product of the mevalonate synthesis pathway, its synthesis can be reduced by interfering with the rate-limiting enzyme, 3-hydroxy-3-methyl-glutaryl-coenzyme A reductase (HMGCoA reductase). Statins, designed as high-affinity HMG-

\footnotetext{
Received September 28, 2019; accepted January 15, 2020; First published February 18, 2020.

The authors declare no competing financial interests.

Author contributions: M.J.O. performed the statistical analysis. Y.E. wrote the initial draft of the paper. M.J.O, L.C.K., and Y.E. contributed to the final version of the paper.

Acknowledgements: We thank Steven Kushner for fruitful discussions and critical reading of the manuscript.

Correspondence should be addressed to Ype Elgersma at y.elgersma@ erasmusmc.nl.

https://doi.org/10.1523/ENEURO.0392-19.2020

Copyright (C) 2020 Ottenhoff et al.

This is an open-access article distributed under the terms of the Creative Commons Attribution 4.0 International license, which permits unrestricted use, distribution and reproduction in any medium provided that the original work is properly attributed.
}

CoA reductase inhibitors, are commonly prescribed for hypercholesterolemia. Over the past several decades, various types of statins have been extensively investigated as potential cancer therapeutics using cellular models, mouse studies, and human clinical trials (Gazzerro et al., 2012; Pisanti et al., 2014; Sopková et al., 2017). On the basis of these findings, Alcino Silva and colleagues explored whether statins might have efficacy in the treatment of RASopathies, a group of neurodevelopmental disorders resulting from mutations that lead to overactivation of the RAS/extracellular signal-regulated kinase (ERK) signaling pathway. Specifically, it has been shown that lovastatin can ameliorate the cognitive deficits in animal models of neurofibromatosis type 1 (Nf1 mice) and Noonan syndrome [Ptpn11 (protein tyrosine phosphatase non-receptor type 11) mice; Li et al., 2005; Lee et al., 2014], although it failed to rescue the deficits in a mouse model of Costello syndrome (Hras mice; Schreiber et al., 2017).

To translate the mouse findings to the clinic, statins were tested in several randomized placebo-controlled trials aimed at improving cognitive function (Krab et al., 2008; Van Der Vaart et al., 2013; Bearden et al., 2016; Payne et al., 2016; Moazen-Zadeh et al., 2018; Stivaros et 
al., 2018). These trials used either the first commerciallyavailable statin, lovastatin, or a second generation statin, simvastatin, that is highly similar in structure and pharmacokinetics to lovastatin (Neuvonen et al., 2008; Gazzerro et al., 2012). Notably, simvastatin has not been used in animal models of the RASopathies, but like lovastatin, simvastatin has been shown to decrease ERK signaling in cultured cells (Fürst et al., 2002; Guillén et al., 2004; Miura et al., 2004; Ghittoni et al. 2005, 2006; Khanzada et al., 2006; Ogunwobi and Beales, 2008; Sundararaj et al., 2008; Kang et al., 2009), as well as in vivo (Chen et al., 2010; Lee et al., 2011; Takayama et al., 2011), including the brain (Ghosh et al., 2009). Simvastatin has a 2- to 4-fold increased potency against HMG-CoA reductase and a higher blood-brain barrier permeability compared with lovastatin (van Vliet et al., 1996; Sierra et al., 2011; Gazzerro et al., 2012; Fong, 2014). These comparative properties of simvastatin and lovastatin might suggest at minimum the non-inferiority of simvastatin versus lovastatin. Although both lovastatin (Mainberger et al., 2013; Bearden et al., 2016) and simvastatin (Stivaros et al., 2018) showed some potential benefits in smaller trials for $\mathrm{NF1}$, three independent large randomized controlled trials of cognition and behavior in children with NF1 using a dose of $40 \mathrm{mg} / \mathrm{d}$ of simvastatin (Krab et al., 2008; Van Der Vaart et al., 2013) or lovastatin (Payne et al., 2016) failed to show efficacy in the primary outcome measures, even when treatment was administered for one year (Van Der Vaart et al., 2013). Thus, no benefits (nor meaningful differences) have been observed between simvastatin and lovastatin in treating NF1-associated cognitive dysfunction. The only sufficiently powered trial that has suggested a benefit for statin treatment on behavior came from a recent study on children with ASD, in which simvastatin was used adjunctively, which yielded a significant decrease of irritability and hyperactivity, but no improvement on three other scales of a behavioral checklist (Moazen-Zadeh et al., 2018).

In light of these mostly negative findings, it is crucial to try to understand why these clinical trials failed. For that, more research is required, and the study by Muscas et al. (2019) is a very important step in that direction. In this study, the authors compared lovastatin with simvastatin treatment in an animal model of fragile X ( $F m r 1$ (fragile $X$ mental retardation) mice). Although ERK signaling in Fmr1 mice is not increased under baseline conditions, it has been shown that the ERK pathway in these mice is hypersensitive and contributes to the excessive protein synthesis which is considered one of the core mechanisms underlying fragile $X$ syndrome pathophysiology (Osterweil et al., 2010). Moreover, lovastatin treatment rescues the ERK-dependent increased of protein synthesis as well as the sensitivity to audiogenic seizures of Fmr1 animals (Osterweil et al., 2013). Given that simvastatin is a more potent inhibitor of HMG-CoA reductase than lovastatin, one would expect that simvastatin treatment would result in a better, or at least a similar rescue. However, in the recent study, Muscas et al. (2019) surprisingly concluded that lovastatin is superior over simvastatin in reducing ERK activation, as well as in its ability to rescue the downstream phenotypes of ERK activation: increased protein synthesis and sensitivity to audiogenic seizures. Therefore, the authors caution against the assumption that simvastatin is a suitable substitute for lovastatin with respect to the treatment of fragile $\mathrm{X}$ or other neurodevelopmental disorders.

If correct, this conclusion would have far reaching implications. Given the increased potency of simvastatin to reduce HMG-CoA reductase, it would suggest that the previously demonstrated rescue of RASopathy phenotypes by statins is not mediated by attenuation of RAS farnesylation but rather through an unknown mechanism that is absent or less potent for simvastatin. This would have considerable impact in the design of potential future clinical trials for treatment of cognitive deficits in RAS related disorders. However, in reviewing the study of Muscas et al. (2019), the question arises whether the study truly represents a side-by-side comparison that warrants such a strong conclusion. Most notable, there is no experiment in which lovastatin and simvastatin are compared at the same dose (and with the same vehicle). In addition, a statistical analysis that would enable a direct comparison of lovastatin and simvastatin is lacking.

Given the aforementioned large body of literature that shows that simvastatin can reduce RAS/ERK signaling in cultured cells as well as in vivo, the finding by Muscas and colleagues, that simvastatin (in contrast to lovastatin) fails to reduce ERK signaling in brain slices, is quite remarkable. However, it is important to note that the investigators used $50 \mu \mathrm{m}$ lovastatin but a 100- to 500 -fold lower dose of simvastatin (the maximum used simvastatin dose is $0.5 \mu \mathrm{M})$. Importantly, the authors previously showed that a lovastatin dose of $10 \mu \mathrm{m}$ is not effective in this particular assay (Osterweil et al., 2013), hence, the failure of simvastatin to reduce ERK activation at doses far below that is not entirely surprising.

For the protein synthesis experiments (which is sensitive to increased ERK signaling), the investigators used again a much lower dose of simvastatin (10- to 500-fold lower) compared with lovastatin (the maximum used simvastatin dose is $5 \mu \mathrm{m}$ ). The lack of efficacy at such a low dose of simvastatin is again not entirely surprising, as the authors previously showed that the lovastatin dose needs to exceed at least $10 \mu \mathrm{m}$ to be effective in this assay (Osterweil et al., 2013). An elegant study by Tuckow et al. (2011) showed that $10 \mu \mathrm{m}$ simvastatin is indeed able to reduce protein synthesis in a mevalonate dependent way, which indicates that at this dose (and under these conditions) there is a clear HMG-CoA-dependent effect of simvastatin on protein synthesis.

The most surprising finding of the study by Muscas and colleagues is the finding that simvastatin treatment at low dose actually worsened the Fmr1 phenotype by further increasing protein synthesis rates. This effect was found to be independent of ERK signaling. This aspect of the study is not only a noteworthy finding, it is also a very worrisome finding with respect to fragile $X$ clinical trials, where the overarching goal is to use statins to reduce protein synthesis and thereby rescue the behavioral phenotypes (Çaku et al., 2014). For the follow-up of these trials it 
would be of great importance to know if a comparable (low) dose of lovastatin (below the dose needed to inhibit ERK) would have a similar negative effect on this phenotype, especially since the dose that can be safely used in clinical trials is much lower than the in vivo dose used in this study.

Whereas in the large, placebo-controlled clinical trials lovastatin was used at the same dose as simvastatin, Muscas and colleagues used a 2- to 30-fold lower dose of simvastin than the dose used for lovastatin $(100 \mathrm{mg} / \mathrm{kg})$ for their in vivo epilepsy experiments. Importantly, the authors previously showed that reducing the lovastatin dose to $30 \mathrm{mg} / \mathrm{kg}$, only rescues the seizure phenotype of $F m r 1$ mice in certain mouse strains (i.e., inbred C57BL/6; Osterweil et al., 2013), indicating that also for lovastatin a lower dose than $100 \mathrm{mg} / \mathrm{kg}$ may not always be effective in this assay.

Beside these differences in dosing, it is questionable if the overall experimental design justifies the conclusion that lovastatin is superior over simvastatin to rescue the core phenotypes of Fmr1 mice. If the ultimate goal of the study is to directly compare two drugs with each other, the drugs should not only be tested side-by-side as interleaved experiments, they should also directly be compared with each other using a statistical analysis that tests for a main effect of treatment, and if significant, followed by a post hoc analysis to compare the drugs. That this can have a substantial effect on the conclusion, can be illustrated by reanalysis of the dichotomous audiogenic seizure data from the paper of Muscas et al. (2019). Performing such analysis using a logistic regression model, reveals that there is a significant main effect of genotype $\left(\chi^{2}(4)=51 ; p<0.0001\right)$, no effect of vehicle $\left(\chi^{2}(2)=0.3 ; p=0.9\right)$ and no interaction of vehicle and genotype $\left(\chi^{2}(1)=0.2 ; p=0.7\right)$. These are important control measures since different concentrations of DMSO solvent were used for each drug and could potentially affect the outcome on seizures (Carletti et al., 2013). This analysis further shows a trend for a main effect of treatment $\left(\chi^{2}(6)=12 ; p=0.07\right)$, but not for the interaction between genotype and treatment $\left(\chi^{2}(4)=4 ; p=0.3\right)$. When performing a post hoc Tukey's test, neither the Fmr1-lovastatin versus Fmr1 "low dose" of simvastatin $(p=0.96)$ nor the Fmr1-lovastatin versus Fmr1 "high dose" of simvastatin treatment $(p>0.99)$ are significantly different from each other. Hence, despite the fact that the lovastatin dose was 2- to 30-fold higher than simvastatin dose, it does not seem to perform significantly better than simvastatin in this seizure assay.

So how can the lack of efficacy of both lovastatin and simvastatin in prior randomized clinical trials of neurodevelopmental disorders be explained, and what can we learn from pre-clinical studies such as the Muscas et al. (2019) study? We believe that two factors are very important to consider when translating findings in animal models to clinical trials in humans.

The first critical factor is the translation of dosing from mice to men. The dose in which a particular drug rescues a phenotype in animal model does not always translate into a clinically applicable and safe dose in humans

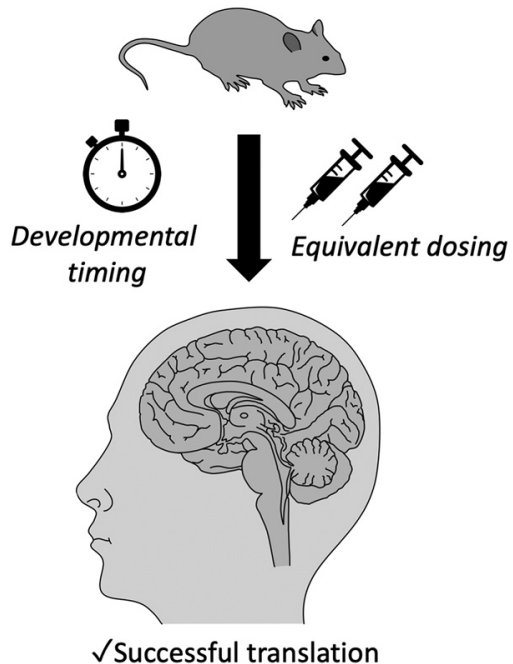

Figure 1. To ensure optimal translation of animal experiments to cognitive clinical trials, it is important that the drug treatment used in animal studies resembles that of clinical trials with respect to equivalent dose (considering also interspecies differences in pharmacodynamics and pharmacokinetics of target tissue), route of administration and drug similarity. Moreover, it is important to take into account the timing of drug administration, as treatment of neurodevelopmental disorders may require intervention during a critical window of development.

(Figure 1). For instance, the study by Muscas et al. (2019) used a lovastatin dose of $100 \mathrm{mg} / \mathrm{kg}$ (intraperitoneal injection) for testing of audiogenic seizures in the fragile $X$ mouse model. This dosing regimen is much higher than needed to inhibit HMG-CoA reductase (Van de Steeg et al., 2013), or the dose used for behavioral rescue in earlier studies of RASopathy mouse models $(10 \mathrm{mg} / \mathrm{kg}$, subcuteanous injection; Li et al., 2005; Lee et al., 2014; Schreiber et al., 2017). More importantly, it is $\sim 100$-fold higher than the equivalent dose used in the clinical trials when also considering bioavailability for oral versus intraperitoneal injection (Zhu et al., 2011; dose conversion calculated by FDA guidelines; www.fda.gov/media/72309/download). Hence, although the partial rescue of audiogenic seizures is of compelling scientific interest, it is important to realize that the direct translational value of such high doses is limited. And although a rescue of behavioral deficits in Fmr1, Nf1, and Ptpn11 animal models has been observed using an oral dose that more closely reflects the dosing used in clinical trials (Li et al., 2005; Osterweil et al., 2013; Lee et al., 2014; Asiminas et al., 2019), it still cannot be excluded that the effective dose of statins in the mouse brain is different from the human brain, as even small species differences in blood brain permeability could eliminate the beneficial effect of statins (Hoshi et al., 2013). This study by Muscas et al. (2019) underscores the importance of looking at effective dosing ranges, and more detailed (in vivo) pharmacological studies in animal models should be performed to elucidate the dose dependency of therapeutic benefit.

The second factor that may affect successful translation to patients is the timing of drug administration. Whereas most pre-clinical studies involved drug treatment of adult animal models of neurodevelopmental 
disorders, it is conceivable that this may not be effective in human patients and that treatment of patients should be started in young children to be maximally effective. Conversely, if a behavioral rescue is observed in young mice (e.g., the rescue of seizures in Fmr1 mice was performed on postnatal day (P)18-P29 mice; Osterweil et al., 2013; Muscas et al., 2019), it is important to investigate if such a rescue is still observed when the brain has fully matured. Interestingly, a recent study in a rat model of fragile $X$ syndrome demonstrated that adult Fmr1 animals no longer exhibited cognitive deficits following brief lovastatin treatment at young age only (Asiminas et al., 2019). Such studies should be further exploited to delineate the precise critical period for optimal treatment of neurodevelopmental disorders (Silva-Santos et al., 2015).

Once these two critical parameters are known for both simvastatin and lovastatin, it may be warranted to consider new clinical trials of statins for treatment of cognition in neurodevelopmental disorders. Hopefully, when using the right conditions, statins will be as effective in humans as they were shown to be in multiple animal models.

\section{References}

Asiminas A, Jackson AD, Louros SR, Till SM, Spano T, Dando O, Bear MF, Chattarji S, Hardingham GE, Osterweil EK, Wyllie DJA, Wood ER, Kind PC (2019) Sustained correction of associative learning deficits after brief, early treatment in a rat model of fragile X syndrome. Sci Transl Med 11:eaao0498.

Bearden CE, Hellemann GS, Rosser T, Montojo C, Jonas R, Enrique N, Pacheco L, Hussain SA, Wu JY, Ho JS, McGough JJ, Sugar CA, Silva AJ (2016) A randomized placebo-controlled lovastatin trial for neurobehavioral function in neurofibromatosis I. Ann Clin Transl Neurol 3:266-279.

Çaku A, Pellerin D, Bouvier P, Riou E, Corbin F (2014) Effect of lovastatin on behavior in children and adults with fragile $X$ syndrome: An open-label study. Am J Med Genet 164:2834- 2842.

Carletti F, Ferraro G, Rizzo V, Cannizzaro C, Sardo P (2013) Antiepileptic effect of dimethyl sulfoxide in a rat model of temporal lobe epilepsy. Neurosci Lett 546:31-35.

Chen YJ, Chen P, Wang HX, Wang T, Chen L, Wang X, Sun BB, Liu DS, Xu D, An J, Wen FQ (2010) Simvastatin attenuates acroleininduced mucin production in rats: Involvement of the Ras/extracellular signal-regulated kinase pathway. Int Immunopharmacol 10:685-693.

Fong CW (2014) Statins in therapy: Understanding their hydrophilicity, lipophilicity, binding to 3-hydroxy-3-methylglutaryl-CoA reductase, ability to cross the blood brain barrier and metabolic stability based on electrostatic molecular orbital studies. Eur J Med Chem 85:661-674.

Fürst J, Haller T, Chwatal S, Wöll E, Dartsch PC, Gschwentner M, Dienstl A, Zwierzina H, Lang F, Paulmichl M, Ritter M (2002) Simvastatin inhibits malignant transformation following expression of the Ha-ras oncogene in NIH 3 T3 fibroblasts. Cell Physiol Biochem 12:19-30.

Gazzerro P, Proto MC, Gangemi G, Malfitano AM, Ciaglia E, Pisanti S, Santoro A, Laezza C, Bifulco M (2012) Pharmacological actions of statins: A critical appraisal in the management of cancer. Pharmacol Rev 64:102-146.

Ghittoni R, Patrussi L, Pirozzi K, Pellegrini M, Lazzerini PE, Capecchi PL, Pasini FL, Baldari CT (2005) Simvastatin inhibits T-cell activation by selectively impairing the function of Ras superfamily GTPases. FASEB J 19:605-607.

Ghittoni R, Napolitani G, Benati D, Ulivieri C, Uliveri C, Patrussi L, Laghi Pasini F, Lanzavecchia A, Baldari CT (2006) Simvastatin inhibits the MHC class II pathway of antigen presentation by impairing Ras superfamily GTPases. Eur J Immunol 36:2885-2893.

Ghosh A, Roy A, Matras J, Brahmachari S, Gendelman HE, Pahan K (2009) Simvastatin inhibits the activation of p21ras and prevents the loss of dopaminergic neurons in a mouse model of Parkinson's disease. J Neurosci 29:13543-13556.

Guillén C, de Gortázar AR, Esbrit P (2004) The interleukin-6/soluble interleukin-6 receptor system induces parathyroid hormone-related protein in human osteoblastic cells. Calcif Tissue Int 75:153159.

Hoshi Y, Uchida Y, Tachikawa M, Inoue T, Ohtsuki S, Terasaki T (2013) Quantitative atlas of blood-brain barrier transporters, receptors, and tight junction proteins in rats and common marmoset. J Pharm Sci 102:3343-3355.

Kang S, Kim ES, Moon A (2009) Simvastatin and lovastatin inhibit breast cell invasion induced by H-Ras. Oncol Rep 21:1317-1322.

Khanzada UK, Pardo OE, Meier C, Downward J, Seckl MJ, Arcaro A (2006) Potent inhibition of small-cell lung cancer cell growth by simvastatin reveals selective functions of Ras isoforms in growth factor signalling. Oncogene 25:877-887.

Krab LC, de Goede-Bolder A, Aarsen FK, Pluijm SMF, Bouman MJ, van der Geest JN, Lequin M, Catsman CE, Arts WFM, Kushner SA, Silva AJ, De Zeeuw Cl, Moll HA, Elgersma Y (2008) Effect of simvastatin on cognitive functioning in children with neurofibromatosis type 1: A randomized controlled trial. JAMA 300:287-294.

Lee J, Lee I, Han B, Park JO, Jang J, Park C, Kang WK (2011) Effect of simvastatin on cetuximab resistance in human colorectal cancer with KRAS mutations. J Natl Cancer Inst 103:674-688.

Lee YS, Ehninger D, Zhou M, Oh JY, Kang M, Kwak C, Ryu HH, Butz D, Araki T, Cai Y, Balaji J, Sano Y, Nam Cl, Kim HK, Kaang BK, Burger C, Neel BG, Silva AJ (2014) Mechanism and treatment for learning and memory deficits in mouse models of Noonan syndrome. Nat Neurosci 17:1736-1743.

Li W, Cui Y, Kushner SA, Brown RAM, Jentsch JD, Frankland PW, Cannon TD, Silva AJ (2005) The HMG-CoA reductase inhibitor lovastatin reverses the learning and attention deficits in a mouse model of neurofibromatosis type 1. Curr Biol 15:1961-1967.

Mainberger $F$, Jung $N$, Zenker $M$, Wahlländer U, Freudenberg L, Langer S, Berweck S, Winkler T, Straube A, Heinen F, Granström S, Mautner V, Lidzba K, Mall V (2013) Lovastatin improves impaired synaptic plasticity and phasic alertness in patients with neurofibromatosis type 1. BMC Neurol 13:131.

Mendola CE, Backer JM (1990) Lovastatin blocks N-ras oncogeneinduced neuronal differentiation. Cell Growth Differ 1:499-502.

Miura SI, Matsuo Y, Saku K (2004) Simvastatin suppresses coronary artery endothelial tube formation by disrupting Ras/Raf/ERK signaling. Atherosclerosis 175:235-243.

Moazen-Zadeh E, Shirzad F, Karkhaneh-Yousefi M-A, Khezri R, Mohammadi M-R, Akhondzadeh S (2018) Simvastatin as an adjunctive therapy to risperidone in treatment of autism: A randomized, double-blind, placebo-controlled clinical trial. J Child Adolesc Psychopharmacol 28:82-89.

Muscas M, Louros SR, Osterweil EK (2019) Lovastatin, not simvastatin, corrects core phenotypes in the fragile $X$ mouse model. eNeuro 6:ENEURO.0097-19.2019.

Neuvonen PJ, Backman JT, Niemi M (2008) Pharmacokinetic comparison of the potential over-the-counter statins simvastatin, lovastatin, fluvastatin and pravastatin. Clin Pharmacokinet 47:463474.

Ogunwobi OO, Beales ILP (2008) Statins inhibit proliferation and induce apoptosis in Barrett's esophageal adenocarcinoma cells. Am J Gastroenterol 103:825-837.

Osterweil E, Krueger D, Reinhold K, Bear M (2010) Hypersensitivity to mGluR5 and ERK1/2 leads to excessive protein synthesis in the hippocampus of a mouse model of fragile X syndrome. J. Neurosci 30:15616-15627.

Osterweil EK, Chuang S-C, Chubykin AA, Sidorov M, Bianchi R, Wong RKS, Bear MF (2013) Lovastatin corrects excess protein synthesis and prevents epileptogenesis in a mouse model of fragile X syndrome. Neuron 77:243-250. 
Payne JM, Barton B, Ullrich NJ, Cantor A, Hearps SJC, Cutter G, Rosser T, Walsh KS, Gioia GA, Wolters PL, Tonsgard J, Schorry E, Viskochil D, Klesse L, Fisher M, Gutmann DH, Silva AJ, Hunter SJ, Rey-Casserly C, Cantor NL, et al. (2016) Randomized placebocontrolled study of lovastatin in children with neurofibromatosis type 1. Neurology 87:2575-2584.

Pisanti S, Picardi P, Ciaglia E, D'Alessandro A, Bifulco M (2014) Novel prospects of statins as therapeutic agents in cancer. Pharmacol Res 88:84-98.

Schreiber J, Grimbergen L-A, Overwater I, Vaart TVD, Stedehouder J, Schuhmacher AJ, Guerra C, Kushner SA, Jaarsma D, Elgersma $Y$ (2017) Mechanisms underlying cognitive deficits in a mouse model for Costello syndrome are distinct from other RASopathy mouse models. Sci Rep 7:1256.

Sebti SM, Tkalcevic GT, Jani JP (1991) Lovastatin, a cholesterol biosynthesis inhibitor, inhibits the growth of human $\mathrm{H}$-ras oncogene transformed cells in nude mice. Cancer Commun 3:141-147.

Sierra S, Ramos MC, Molina P, Esteo C, Vázquez JA, Burgos JS (2011) Statins as neuroprotectants: A comparative in vitro study of lipophilicity, blood-brain-barrier penetration, lowering of brain cholesterol, and decrease of neuron cell death. J Alzheimers Dis 23:307-318

Silva-Santos S, Woerden G, Bruinsma C, Mientjes E, Jolfaei M, Distel B, Kushner S, Elgersma Y (2015) Ube3a reinstatement identifies distinct developmental windows in a murine Angelman syndrome model. J Clin Invest 125:2069-2076.

Sopková J, Vidomanová $E$, Strnádel J, Sõkovierová $H$, Halašová $E$ (2017) The role of statins as therapeutic agents in cancer. Gen Physiol Biophys 36:501-511.

Stivaros S, Garg S, Tziraki M, Cai Y, Thomas O, Mellor J, Morris AA, Jim C, Szumanska-Ryt K, Parkes LM, Haroon HA, Montaldi D, Webb N, Keane J, Castellanos FX, Silva AJ, Huson S, Williams S, Gareth Evans D, Emsley R, et al. (2018) Randomised controlled trial of simvastatin treatment for autism in young children with neurofibromatosis type 1 (SANTA). Mol Autism 9:12.
Sundararaj KP, Samuvel DJ, Li Y, Nareika A, Slate EH, Sanders JJ, Lopes-Virella MF, Huang Y (2008) Simvastatin suppresses LPS-induced MMP-1 expression in U937 mononuclear cells by inhibiting protein isoprenylation-mediated ERK activation. J Leukoc Biol 84:1120-1129.

Takayama N, Kai H, Kudo H, Yasuoka S, Mori T, Anegawa T, Koga M, Kajimoto H, Hirooka Y, Imaizumi T (2011) Simvastatin prevents large blood pressure variability induced aggravation of cardiac hypertrophy in hypertensive rats by inhibiting RhoA/Ras-ERK pathways. Hypertens Res 34:341-347.

Tuckow A, Jefferson S, Kimball S, Jefferson L (2011) Simvastatin represses protein synthesis in the muscle-derived $\mathrm{C}_{2} \mathrm{C}_{12}$ cell line with a concomitant reduction in eukaryotic initiation factor $2 \mathrm{~B}$ expression. Am J Physiol Endocrinol Metab 300:E564-E570.

Van Der Vaart T, Plasschaert E, Rietman AB, Renard M, Oostenbrink $R$, Vogels $A$, de Wit $M-C$, Descheemaeker M-J, Vergouwe $Y$, Catsman-Berrevoets CE, Legius E, Elgersma Y, Moll HA (2013) Simvastatin for cognitive deficits and behavioural problems in patients with neurofibromatosis type 1 (NF1-SIMCODA): A randomised, placebo-controlled trial. Lancet Neurol 12:1076-1083.

van de Steeg E, Kleemann R, Jansen HT, van Duyvenvoorde W, Offerman EH, Wortelboer HM, DeGroot J (2013) Combined analysis of pharmacokinetic and efficacy data of preclinical studies with statins markedly improves translation of drug efficacy to human trials. J Pharmacol Exp Ther 347:635-644.

van Vliet AK, Nègre-Aminou P, van Thiel GC, Bolhuis PA, Cohen LH (1996) Action of lovastatin, simvastatin, and pravastatin on sterol synthesis and their antiproliferative effect in cultured myoblasts from human striated muscle. Biochem Pharmacol 52:1387-1392.

Zhu Y, D'Agostino J, Zhang Q-Y (2011) Role of intestinal cytochrome P450 (P450) in modulating the bioavailability of oral lovastatin: Insights from studies on the intestinal epithelium-specific P450 reductase knockout mouse. Drug Metab Dispos 39:939-943. 